See discussions, stats, and author profiles for this publication at: https://www.researchgate.net/publication/276121052

\title{
Liver X receptor agonist downregulates growth hormone signaling in the liver
}

Article in Hormone molecular biology and clinical investigation · November 2011

DOI: 10.1515/HMBCl.2011.125

\section{CITATIONS}

6

7 authors, including:

Fahad Zadjali

Sultan Qaboos University

46 PUBLICATIONS 524 CITATIONS

SEE PROFILE

Ewa C S Ellis

Karolinska Institutet

150 PUBLICATIONS 4,970 CITATIONS

SEE PROFILE
51

Mercedes Mirecki-Garrido

Universidad de Las Palmas de Gran Canaria

14 PUBLICATIONS 93 CITATIONS

SEE PROFILE

Gunnar Norstedt

Karolinska Institutet

89 PUBLICATIONS 3,065 CITATIONS

SEE PROFILE

Some of the authors of this publication are also working on these related projects:

VALORACIÓN DEL TRATAMIENTO CON SELLANTES BIOLÓGICOS Y TERAPIA CELULAR EN LA PREVENCIÓN DE LA FÍSTULA BRONCOPLEURAL EN UN MODELO EXPERIMENTAL IN VIVO View project

Socs2 project View project 


\section{Liver $\mathrm{X}$ receptor agonist downregulates growth hormone signaling in the liver}

\author{
Fahad Zadjali ${ }^{1-3, *}$, Ruyman Santana-Farre ${ }^{4}$, \\ Mercedes Mirecki-Garrido ${ }^{4}$, Ewa Ellis ${ }^{5}$, \\ Gunnar Norstedt ${ }^{2}$, Leandro Fernandez-Perez ${ }^{4}$ \\ and Amilcar Flores-Morales ${ }^{1}$
}

\author{
${ }^{1}$ Novo Nordisk Foundation Center for Protein Research, \\ Faculty of Health Sciences, University of Copenhagen, \\ Copenhagen, Denmark \\ ${ }^{2}$ Department of Molecular Medicine and Surgery, Karolinska \\ Institutet, Stockholm, Sweden \\ ${ }^{3}$ Department of Biochemistry, College of Medicine and \\ Health Sciences, Sultan Qaboos University, Kodh, Oman \\ ${ }^{4}$ Department of Clinical Sciences-Pharmacology Unit, Faculty \\ of Health Sciences, University of Las Palmas GC, Canary \\ Institute for Cancer Research (ICIC), Las Palmas, Spain \\ ${ }^{5}$ Unit for Transplantation Surgery, Liver Cell Laboratory, \\ Karolinska Institutet, Huddinge, Sweden
}

\begin{abstract}
Liver X receptor (LXR) agonists have been shown to influence the development of hyperlipidemia and atherosclerosis in mouse models. It has also been demonstrated that some LXR agonists can cause hepatic steatosis in experimental animals. Growth hormone (GH) is known to regulate hepatic metabolism and the absence of hepatic GH receptors (GHR) leads to hepatic steatosis. In this study, we analyzed whether the actions of LXR agonists could involve interference with GH signaling. We showed that LXR agonists impair GH signaling in hepatocytes. LXR agonist treatment attenuated GH induction of suppressor of cytokine signaling 2 (SOCS2), SOCS3, and $C I S$ mRNA levels in BRL-4 cells. Likewise, the activity of a luciferase reporter vector driven by the $\mathrm{GH}$ response element (GHRE) of the SOCS2 gene was inhibited by simultaneous treatment with an LXR agonist. The inhibitory effect of LXR agonists on GH signals can be mimicked by overexpression of the LXR regulated factors, sterol regulatory element binding protein 1 (SREBP1) and SREBP2, in hepatic cells. In both cases total and phosphorylated signal transducers and activators of transcription 5b (STAT5b) protein levels were significantly reduced. DNA binding assays demonstrated that SREBP1 binds to an E-box within a previously defined GHRE in the SOCS2 gene promoter, but does not compete with STAT5b binding to a nearby site in the same promoter

*Corresponding author: Fahad Zadjali, Department of Molecular Medicine and Surgery, Karolinska Institutet,

CMM L8:01, 17176 Stockholm, Sweden

Phone: +46 (0) 851773203, E-mail: fahad.al-zadjali@ki.se

Received August 29, 2011; accepted November 9, 2011
\end{abstract}

construct. Taken together, our findings indicate that the inhibitory effects of LXR agonists on GH signaling are mediated by SREBP1, through the downregulation of STAT5b gene transcription and stimulation of STAT5b protein degradation. The findings provide a new insight into the understanding of the molecular actions of LXR agonists, which may be of relevance to their pharmacological actions.

Keywords: 9-cis retinoic acid; growth hormone; lipid; LXR; RXR; SOCS2; T0901317.

\section{Introduction}

The liver $\mathrm{X}$ receptor- $\alpha(\mathrm{LXR} \alpha)$ and $\operatorname{LXR} \beta$ (also known as NR1H3 and NR1H2, respectively), belong to the nuclear receptor superfamily of ligand activated transcription factors [1]. LXRs heterodimerize with the retinoic acid receptor (RXR), to regulate transcription of target genes, upon activation by oxidized derivatives of cholesterol, also known as oxysterols [1]. In the liver, LXR $\alpha$ activation induces a complex transcriptional network involved in the control of intracellular nonesterified cholesterol levels. Experimental studies with synthetic ligands, T0901317 and GW3965, indicate that the pharmacological activation of LXR protects against atherosclerosis, reduces plasma cholesterol levels and improves glucose tolerance in models of type 2 diabetes [2]. Unfortunately, these positive effects are accompanied by hypertriglyceridemia and hepatic steatosis, which presents risks for the development of cardiovascular disease [3]. This raises concerns regarding the application of LXR agonists in the management of common metabolic disorders linked to obesity and type 2 diabetes. A better understanding of the molecular basis for the pleiotropic effects of LXR in the liver, may lead to an improved safety profile for future LXR modulators.

Growth hormone $(\mathrm{GH})$ actions are triggered by its binding to the GH receptor (GHR) and the activation of its associated kinase, JAK2. This, in turn, results in the tyrosine phosphorylation of the transcription factor signal transducers and activators of transcription 5b (STAT5b), which translocates to the nucleus to modulate expression of GH target genes, such as insulin growth factor-1 (IGF-1) and the suppressor of cytokine signaling 2 (SOCS2) [4]. The main metabolic actions of GH are anabolic, promoting protein synthesis in the muscle through the catabolism of fatty acids as the energy source. Because of GH effects on lipid mobilization from the liver, $\mathrm{GH}$ deficient patients and mice where GH signaling is specifically inactivated in the liver [5], are characterized by hepatic steatosis [6]. It is interesting to note that treatment of animals with the LXR agonist T0901317 also causes severe hepatic steatosis [3] and LXR $\alpha / \beta$ 
knockout mice $\left(\mathrm{LXR}^{-/}\right)$are resistant to high fat diet induced hepatic steatosis [7, 8]. Interestingly, in this model, the levels of hepatic type 2 deiodinase mRNA, which is under GH control, were elevated [7]. LXR and GHR activations in the liver also lead to antagonizing effects on glucose metabolism. LXR agonists show an antidiabetic effect by inhibition of gluconeogenesis and reduction of the expression of phosphoenolpyruvate carboxykinase (PEPCK) and glucose-6-phosphatase [2], while GHR activation induces hepatic gluconeogenesis and increases the mRNA levels of gluconeogenic enzymes [9].

The key regulatory role of $\mathrm{GH}$ on liver lipid metabolism and insulin sensitivity opens the question of whether some of the physiological actions that are attributed to the LXR ligands could be explained by interference with GH signaling. We performed this study to provide new insights into the relationship between LXR and GHR signaling in hepatocytes.

\section{Materials and methods}

\section{Materials}

Recombinant rat GH was from the National Hormone and Pituitary Program, National Institutes of Health (NIH, Bethesda, MD, USA) and recombinant human GH was from Novo Nordisk A/S (Gentofte, Denmark). Oligonucleotides were obtained from ThermoElectron (Bremen, Germany). T0901317 and GW3965 (LXR agonists), 9-cis retinoic acid (RXR agonist) and cycloheximide (CHX) were purchased from Sigma-Aldrich (St. Louis, MO, USA).

\section{Plasmid cloning and site-directed mutagenesis}

The luciferase reporter vector containing the GHRE (696bp) in the first intron of SOCS2 human gene, named P5, has been previously described [10]. The fatty acid synthase (FAS) promoter constructs (pFAS) were a generous gift of Dr. T. Osborne [11] and the promoter construct of ATP-binding cassette (pABCA1) was a gift from Dr. Castrillo A [12]. Myc-SREBP1a and myc-SREBP2 (sterol regulatory element binding protein) expressing vectors were a generous gift from Dr. J. Ericsson [13].

\section{Cell culture and transfection assays}

Buffalo rat liver (BRL) cells stably transfected with the rat GHR, designated BRL-4 cells, were cultured as described [10]. Primary human hepatocytes were isolated from discarded organ-donor tissue. Isolated hepatocytes were obtained from the liver cell laboratory, KICLINTEC, Huddinge University Hospital. Ethics approval to use hepatocytes was given by the local Ethical Committee, Stockholm (DNr: 2010/678-31/3). Cells were maintained in Williams E medium as described previously [14]. DNA plasmid transfections were carried as recommended by the manufacturer's instructions on 70\%-80\% confluent cells using lipofectamine with Plus reagent (Invitrogen, Carlsbad, CA, USA). Cells were treated with $1 \mu \mathrm{M}$ LXR agonists, $10 \mu \mathrm{M}$ RXR agonist or $0.1 \%$ DMSO as vehicle control and with GH $50 \mathrm{nM}$.

\section{Reporter gene assay}

pSOCS2-TK-LUC ( $1 \mathrm{ug}$ ) was co-transfected with 10 or $50 \mathrm{ng}$ of the nuclear form of SREBP1a, SREBP2 or pCDNA3 (Invitrogen), and $50 \mathrm{ng} \beta$-galactosidase reporter plasmid. Medium was replaced with serum-free DMEM 16-18 h posttransfection, and cells were treated with $\mathrm{rGH}$ and harvested $36 \mathrm{~h}$ after transfection in reporter lysis buffer for luciferase assays (Promega, Madison, WI, USA). Transfection experiments were performed at least three times.

\section{Analysis of gene transcription}

Total RNA was isolated from treated cells using the RNeasy Mini Kit (Qiagen, Valencia, CA, USA). cDNA synthesis and quantitative realtime PCR were performed as described previously [10]. Expression levels of genes were normalized to those of ribosomal RNA S18 or cyclophilin genes.

\section{Cell lysis and immunoblotting}

Cells were treated as described in the figure legends. Protein extraction and SDS-PAGE were performed as described previously [10]. Antibodies against STAT5, pSTAT5 (Tyr694) and SOCS2 were purchased from Cell Signaling Technology (Frankfurt A. M., Germany) and, SREBP1, SREBP2, $\beta$-actin, myc and GHR antibodies were purchased from Santa Cruz Biotechnology (Beverly, MA, USA).

\section{CHX chase experiments}

BRL-4 cells were transfected with myc-SREBP1a, myc-SREBP2 or control plasmid. Cells were split into different plates for treatment with $100 \mu \mathrm{g} / \mathrm{mL}$ CHX (Sigma-Aldrich) $24 \mathrm{~h}$ post-transfection and then lysed at different time points for protein extraction.

\section{ABCD assay (avidin, biotin, complex, DNA)}

In this assay, protein-DNA complexes were immobilized by biotinylated oligonucleotides to a streptavidin matrix. BRL-4 cells were treated with LXR/RXR agonists for $48 \mathrm{~h}$ and then stimulated with $\mathrm{GH}$ for $10 \mathrm{~min}$, before lysis in $50 \mathrm{mM}$ HEPES $\mathrm{pH} 7.5,150 \mathrm{mM} \mathrm{KCl}, 1 \mathrm{mM}$ EGTA, 1\% NP-40, $10 \%$ glycerol, $1.5 \mathrm{mM} \mathrm{MgCl}_{2}, 1 \mathrm{mM} \mathrm{Na}_{3} \mathrm{VO}_{4}, 1 \mathrm{X}$ cocktail inhibitor, $50 \mathrm{mM} \mathrm{NaF}$ and $1 \mathrm{mM}$ PMSF. BRL4 cell extracts $(250 \mu \mathrm{g})$ were incubated with $2 \mu \mathrm{g}$ biotinylated oligonucleotide and $5 \mu \mathrm{g}$ salmon sperm DNA (Stratagene, La Jolla, CA, USA). Mixtures were incubated overnight at $4^{\circ} \mathrm{C}$ under constant rotation. Then $40 \mu \mathrm{L}$ equilibrated streptavidin agarose beads (Amersham, Arlington Heights, IL, USA) were added to the mix and further incubated for $2 \mathrm{~h}$ at $4{ }^{\circ} \mathrm{C}$ on a rotator. After centrifugation, beads were washed repeatedly with lysis buffer containing $50 \mathrm{mM} \mathrm{KCl}$, then boiled in sample buffer and separated by SDS-PAGE. SREBP1 and STAT5b bindings were detected by Western blot. We also repeated the ABCD assay using extracts from separately treated cells after $48 \mathrm{~h}$ treatment with LXR/ RXR agonist or after 10 min treatment with GH. Equal amounts of total protein from each cell lysate were used for the assay. As control oligonucleotides for SREBP1 and STAT5b binding, we used sterol response element (SRE) element in the acetyl-CoA carboxylase (ACC1) genes and GHRE, containing two STAT5b sites, in SPI-2.1 gene. 3' Biotinylated oligonucleotides were ordered from ThermoElectron (Bremen, Germany) with the following forward oligonucleotides sequences: WT: 5'-ccegcg-gtcacgtgaggccgattcetggaaagttcctggaaagcc g-3', mut-Ebox: 5'-cccgcggtgtcgtgaggccgatt-cetggaaagttcctggaaagcc g-3', mut-STAT5b: 5'-cccgcggtcacgtgaggccgaaacctggaaagaacc tggaaagcc g-3', mut-Ebox/STAT5b: $5^{\prime}$-ccc cggtgtcgtgaggecgaaacctggaaagaacctggaaa gccg-3', SRE: 5'-tcgcatcacaccaccgcg g-3', GHRE: $5^{\prime}$-tcgacgcttctactaatcccatgttctgag aaatcatccag- $3^{\prime}$. The forward and complementary oligonucleotides were heated for $10 \mathrm{~min}$ at $95^{\circ} \mathrm{C}$ then cooled down to anneal at room temperature. 

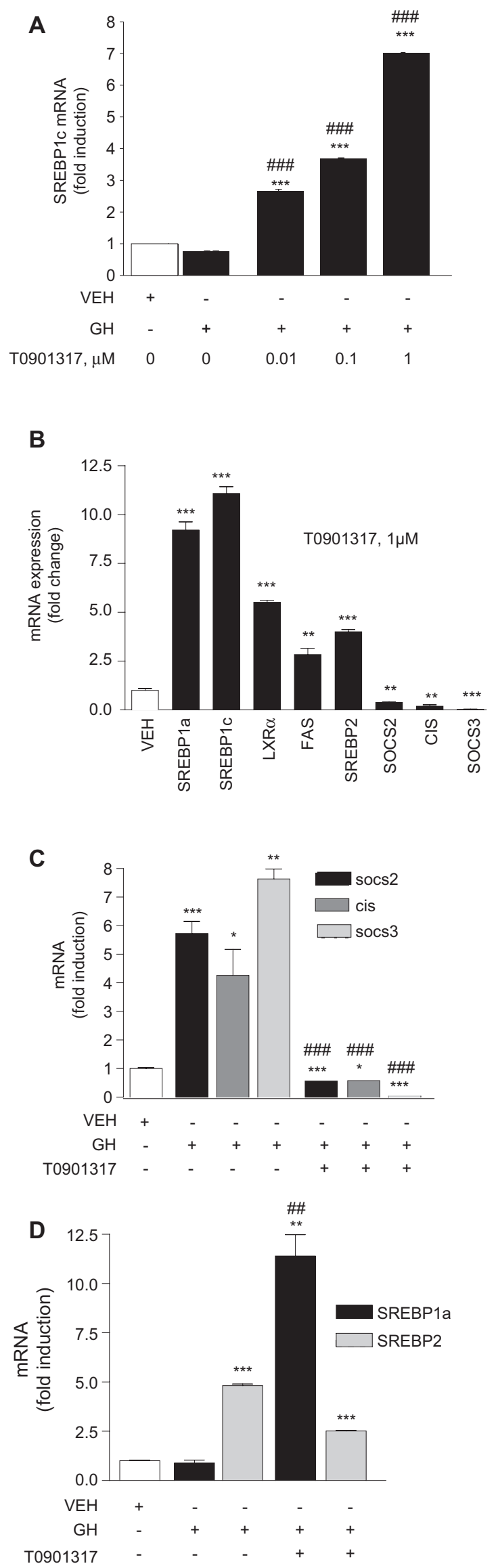

Figure 1 Effects of T0901317 on GH-dependent expression of SOCS 2 mRNA in BRL-4 cells. (A) BRL-4 cells were treated for $4 \mathrm{~h}$ with different doses of LXR agonist (T0901317) or vehicle (VEH) then followed with $2 \mathrm{~h}$ rGH stimulation before quantification of gene expression, normalized to cyclophiline gene expression. (B) BRL-4 cells were treated for $40 \mathrm{~h}$ with $1 \mu \mathrm{M}$ LXR agonist (T0901317) followed by quantification of gene expression. (C)+(D) BRL-4 cells were treated similarly as (B) then followed with rGH (50 nM) stimulation for $2 \mathrm{~h}$ before quantification of gene expression. $\mathrm{VEH}=0.1 \%$ $\mathrm{DMSO}, \mathrm{Me}_{2} \mathrm{SO}$. Values are expressed as means $\pm \mathrm{SEM}$.

*Indicates significant differences compared to GH-unstimulated cells, \# indicates significant differences to cells treated with GH alone.

$* * \mathrm{p}<0.01, * * * \mathrm{p}<0.001$

\section{Statistical analysis}

Statistically significant differences were assessed with the two-tailed Student's t-test. All statistical analysis was performed using SPSS for Windows (version 10.0, Chicago, IL, USA). Statistical significance was reported for $\mathrm{p}<0.05$.

\section{Results}

\section{GH induced gene expression is inhibited by LXR agonist}

We used BRL cells stably transfected with the rGHR (BRL-4) [15] to analyze GH signals. These cells express endogenous JAK2 and STAT5b and respond to GH with the transcriptional induction of STAT5b-regulated genes, such as CIS and SOCS2, although they express little $I G F-1$ [15]. We first performed a dose-response experiment in BRL-4 cells to assess their response to T0901317, by measuring changes in SREBP1C expression (Figure 1A). Maximal activity was observed at $1 \mu \mathrm{M}$, thus this was chosen as the working concentration for the following experiments. In order to understand the interaction between LXR and GH signaling, we studied the effect of the LXR synthetic ligand T0901317 on the mRNA levels of selected genes. As shown in Figure 1B, $40 \mathrm{~h}$ treatment of BRL-4 cells with the LXR agonist resulted in significantly increased mRNA levels of $L X R \alpha, S R E B P 1 a$ and $S R E B P 2$, as well as its downstream target fatty acid synthase $(F A S)$ gene. In addition, we observed a marked downregulation of GH-regulated genes, SOCS2, SOCS3 and CIS. We further studied the LXR agonist effect after $2 \mathrm{~h}$ stimulation in the presence or absence of $\mathrm{GH}$. As expected, $\mathrm{GH}$ has a significant stimulatory activity on the expression of SOCS2, SOCS3 and cytokine-induced SH2 protein (CIS) (Figure 1C). It also increased $S R E B P 2$ expression but failed to stimulate the expression of SREBPIa (Figure 1D). In a combined treatment with the LXR agonist, significant inhibition of GH induced gene expression was detected (Figure 1C).

LXRs form heterodimeric complexes with RXRs [1]. Hence, we examined the effect of short-term treatment (4 h) with the RXR ligand, 9-cis retinoic acid, alone or in combination with the LXR agonist, in the regulation of GH induction of SOCS2 gene expression. Treatment of BRL-4 cells with GH resulted in a 14-fold increase in the levels of SOCS 2 mRNA while $4 \mathrm{~h}$ pretreatment with the RXR and 
LXR agonist alone or in combination resulted in a moderate induction of SOCS2 mRNA levels (Figure 2A). Individual pretreatment with each of the agonists did not have an effect on GH-stimulated SOCS2 expression. In contrast, the combined pretreatment resulted in a significant inhibition of the GH effect parallel to maximal induction of SREBPIc (Figure 2B). Together, these data demonstrate that GH-dependent gene expression is negatively regulated by LXR activation in hepatic cells.

\section{LXR agonist inhibits STAT5b activation by GH}

We have previously identified two evolutionary conserved cis GAS-like elements (TTCNNNAGG) in the SOCS2 gene that bind to STAT5b and mediate GH transcriptional activity [10]. In order to better understand the effects of the LXR ligand T0901317 on GH signaling, we analyzed the activity of a luciferase reporter gene driven by the GHRE identified in the SOCS2 promoter [10]. As controls, we used promoters of LXR responsive genes: $A B C A 1$ transporter (pABCA1) and $F A S$ (pFAS). The latter contains an SREBP1 binding site that indirectly mediates LXR actions. As shown in Figure 2C, GH stimulates the luciferase activity of pSOCS2 while 6 and $24 \mathrm{~h}$ treatment with the LXR agonist resulted in inhibition of this effect, while stimulating its targets pABCA1 and pFAS. This finding suggests that the mechanisms of LXR mediated inhibition of GH signaling involve the regulation of STAT5b activity.

Having demonstrated the antagonistic effect of the LXR ligand T0901317 on STAT5b mediated transcription, we next analyzed its effects on GHR signaling. Firstly, we analyzed GH activation of STAT5b in BRL-4 cells treated with the LXR and RXR agonists, alone or in combination. As observed in Figure 3A, GH treatment increased STAT5b phosphorylation, but had minor effects on SREBP1 protein precursor levels. On the other hand, treatment with either the LXR or RXR agonist did not alter basal STAT5b phosphorylation levels, but led to an increased level of SREBP1, an effect that was further enhanced by the combined treatment. In this setup, pretreatment with either the RXR or LXR agonist, or the combination of both, significantly decreased the levels of STAT5b in the presence of GH, while the amount of active (phosphorylated) STAT5b was only significantly affected by the combined treatment. In order to explore the wider relevance of these findings, we analyzed the effects of LXR and RXR agonists in human primary hepatocytes, which express high GH receptor levels endogenously. As shown in Figure 3B, treatment with LXR and RXR agonists enhanced the expression of premature and mature forms of SREBP1 protein. After 30 min of GH treatment, we observed reduced levels of total and phosphorylated STAT5b as well as GHR in cells treated with the LXR and RXR agonists, as compared with cells treated with vehicle control. To confirm our findings, we analyzed the effects of another LXRspecific, but weaker, agonist GW3965 [16]. As can be seen in Figure 3C, GW3965 treatment of BRL-4 cells increased SREBP1 expression and decreased STAT5b protein levels, similar to the effects observed for T0901317.
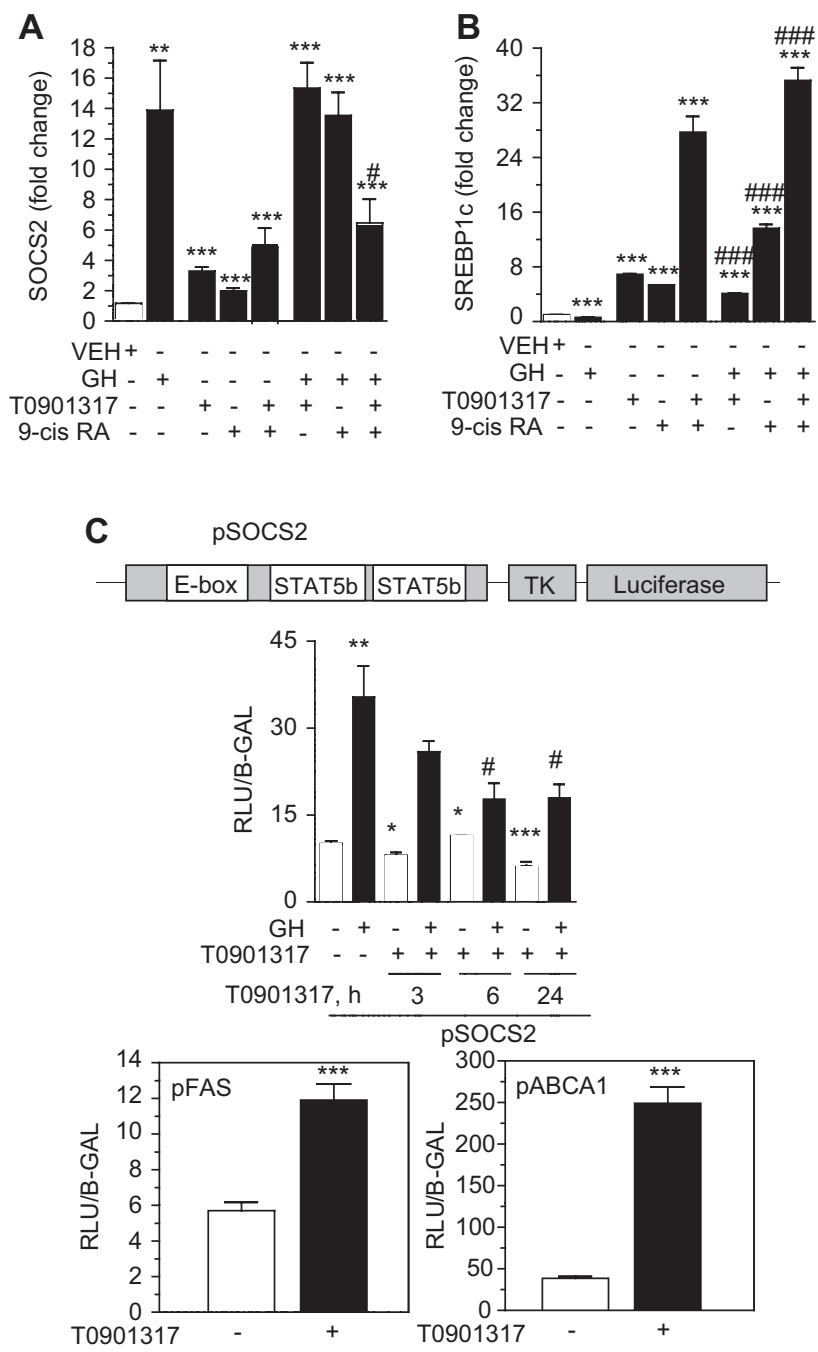

Figure 2 LXR and RXR agonist represses GH-dependent transcriptional activation of SOCS-2. (A, B) Expression of SOCS2 and SREBP1c was measured after $4 \mathrm{~h}$ treatment with LXR (T0901317), RXR (9-cis RA) agonists or a combination of both. The relative expression levels were scaled to the vehicle-matched control group. (C) Luciferase activity of SOCS2 promoter (pSOCS2) from BRL-4 cells after treatment with LXR agonist (T0901317) for 3, 6, and $24 \mathrm{~h}$ with or without $12 \mathrm{~h}$ stimulation with rGH. Transfection of plasmids pFAS (marker of SREBP1 activity) and pABCA1 (marker of SREBP2 activity) were used as positive controls for LXR agonist treatment. Data shown are means \pm SD.

*Indicates significant differences compared to GH-unstimulated cells, \# indicates significant differences to cells treated with GH alone. $* \mathrm{p}<0.05, * * \mathrm{p}<0.01, * * * \mathrm{p}<0.001$.

\section{SREBP-1 downregulates SOCS2 promoter activity}

The effects of the LXR ligand T0901317 on GH induction of SOCS2 expression, coincide with the elevated expression of SREBP1a, suggesting the involvement of this transcription factor in the negative regulation of SOCS2 gene transcription at the promoter level. Therefore, we tested the influence of SREBP1a overexpression on the activity of the SOCS2 promoter. The luciferase-driven SOCS2 promoter was transiently co-transfected in BRL-4 cells, with different concentrations 
A

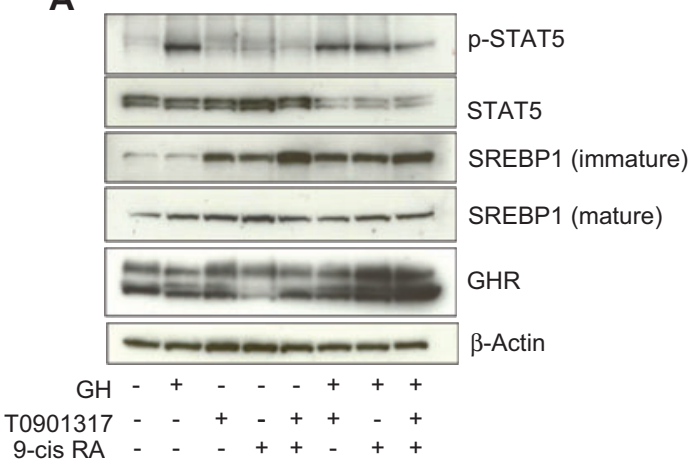

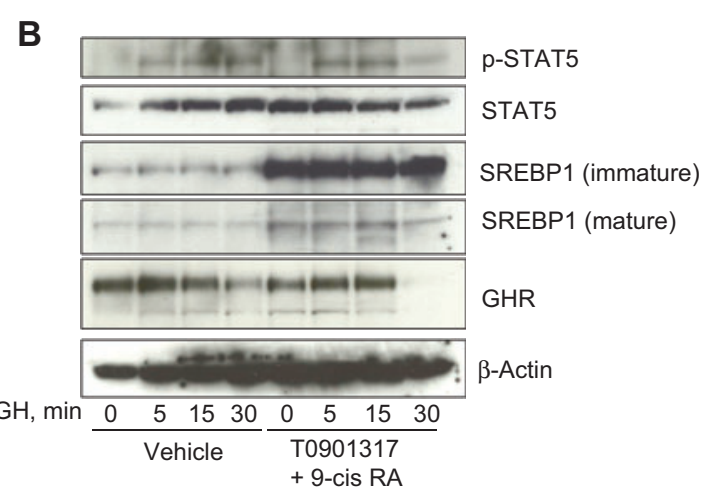

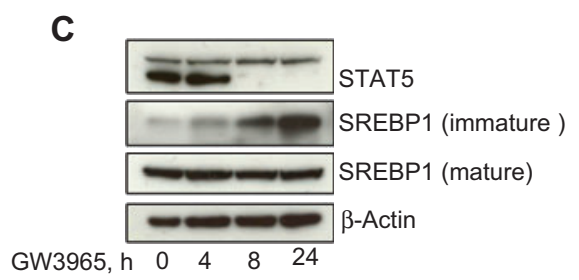

Figure 3 LXR activation downregulates STAT5b in primary hepatocytes and in cultured liver cells. (A) Western blot of protein extracts from BRL-4 cells treated for $8 \mathrm{~h}$ with $1 \mu \mathrm{M}$ LXR agonist (T0901317) and $10 \mu \mathrm{M}$ RXR, with or without $4 \mathrm{~h}$ stimulation with rGH. (B) Primary human hepatocytes were treated for $16 \mathrm{~h}$ with LXR (T0901317) and RXR (9-cis RA) agonists followed by GH stimulation as indicated. (C) BRL-4 cells were treated with $1 \mu \mathrm{M}$ LXR agonist, GW3965, at different time points.

of a plasmid encoding the active nuclear form of SREBP1a. As a control experiment, transfection with SREBP1a plasmid induced the promoter activity of FAS gene (pFAS) (Figure 4A). We observed a significant downregulation of SOCS2 promoter activity after co-transfection with 10 and 50 ng of SREBP-1a (90\% and 92\% downregulation, respectively), (Figure 4B). These data mimic the findings observed in Figure 1A, where LXR agonist treatment of BRL-4 cells resulted in reduced SOCS2 expression, coinciding with enhanced expression of SREBPIa.

A highly conserved putative SREBP1 binding site, E-box, CANNTG, exists 13 bp upstream of the STAT5b binding site in the GHRE found in the SOCS2 gene promoter [10], Figure 4C. Thus, the inhibitory effect of SREBP1 on GH-induced SOCS2 expression, could potentially be explained by a sterical hindrance of STAT5b binding to the promoter. To analyze this possibility, we performed protein-DNA binding assays using biotinylated DNA fragments of the SOCS2 promoter carrying the E-box and the STAT5b binding sites (Wild type, WT), or the mutant version of E-box and STAT5b. We used two oligonucleotide fragments as positive controls for SREBP1 and STAT5b DNA binding activity (Figures 4C, D). The first consists of a DNA fragment containing an SREBP binding site (SRE) found in the $A C C l$ gene promoter [17]. The second control consists of a DNA fragment containing two STAT5b binding sites (GHRE) found in the SPI-2.1 rat gene promoter [18]. Treatment with LXR and RXR agonists increased the SREBP1 protein levels in the total cell lysates and accordingly enhanced the binding to the WT and STAT5b mutated fragments, but not to the E-box mutated construct. This finding demonstrates that SREBP1 can bind to the SOCS 2 promoter. On the other hand, STAT5b binding to the WT and E-box mutated DNA fragment was enhanced by
GH stimulation. However, the binding was attenuated when cells were co-treated with LXR and RXR agonists, coinciding with the reduction in the total STAT5b levels detected in the total cell lysates. In an additional analysis, we treated BRL-4 cells with the LXR/RXR agonist and GH separately, to avoid the LXR mediated downregulation of STAT5b protein levels. Lysates from independently treated cells were mixed in a 1:1 ratio and incubated with DNA fragments as shown in Figure 4C. The mixed lysates of cells independently treated with GH or T0901317 exhibited similar STAT5b protein levels, Figure 4D. The binding of STAT5b to the WT construct was enhanced in the samples stimulated with GH and the binding was not affected by co-incubation with LXR/RXR agonist treated lysates, which exhibited high levels of SRBEP1 binding activity. This finding does not support the model where SOCS 2 downregulation by LXR agonists is due to a competition between STAT5 and SREBP1 for their respective binding to the SOCS2 promoter. It adds support to the hypothesis that inhibition of SOCS2 promoter activity upon LXR activation is due to downregulation of STAT5b protein levels.

\section{SREBP1 downregulates STAT5b protein levels}

To further elucidate the possible mechanisms whereby an LXR agonist mediated reduction of STAT5b, we analyzed STAT5b protein levels in BRL-4 cells overexpressing SREBP1a or SREBP2. In Figure 5A, we showed that these cells exhibit a significant reduction in both total and active phosphorylated STAT5 protein levels as compared to controls. To investigate if the reduction of STAT5b levels by LXR results from posttranslational instability, we treated BRL-4 cells with CHX and followed the degradation of STAT5b. As shown in Figure 5B, a faster decrease of STAT5b proteins levels after CHX 
A

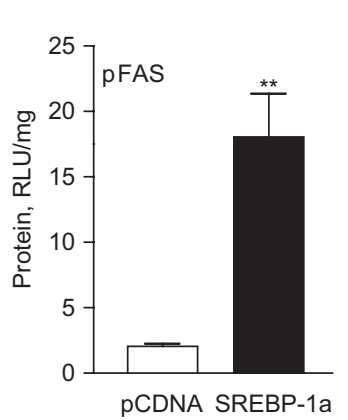

B

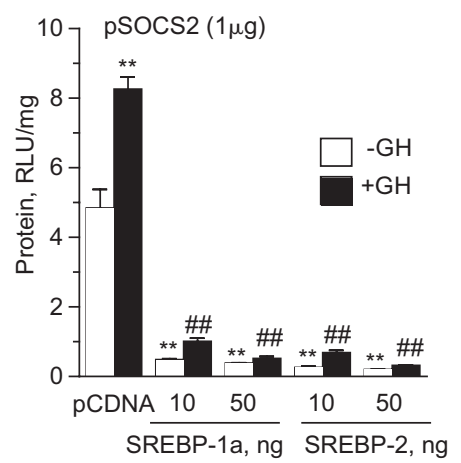

C

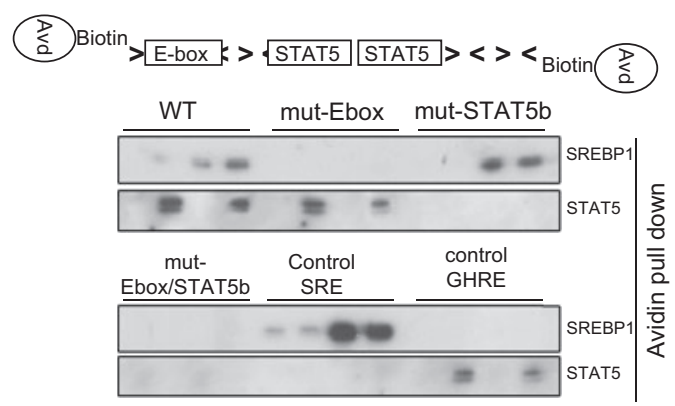

$\mathrm{GH}-+-++++++$

LXR/RXR - - + + - + + - - + +
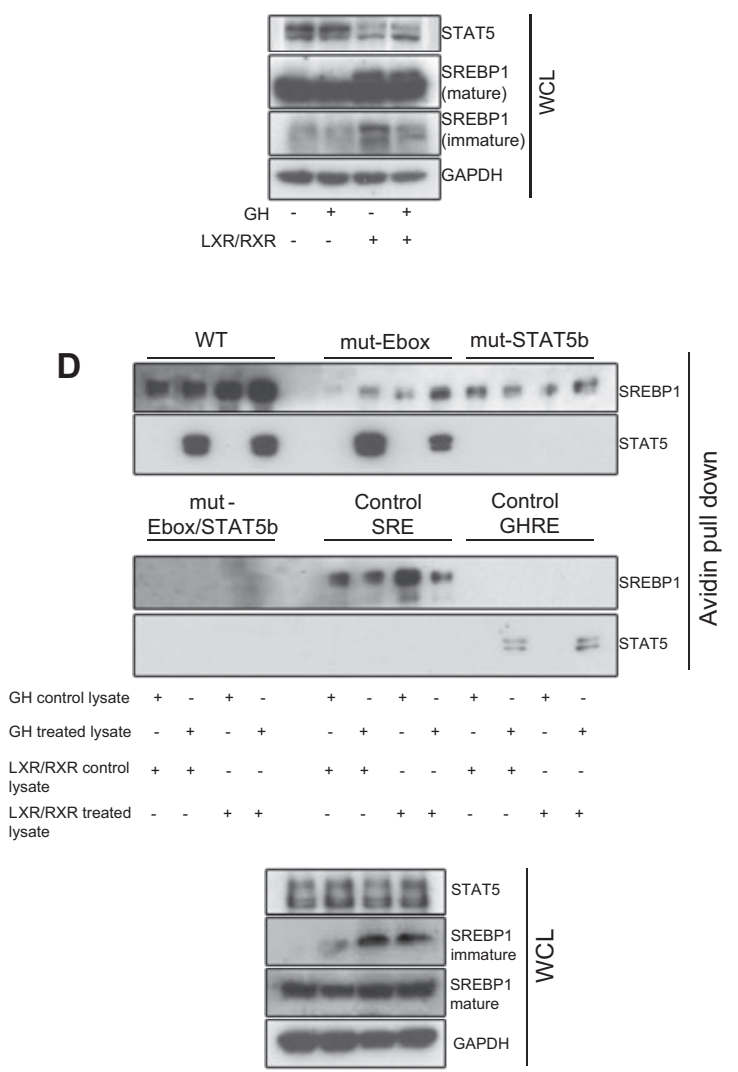

$\mathrm{GH}$ control lysate + ++

$\mathrm{GH}$ treated lysate - + + +

LXR/RXR control + + - -

lysate

LXR/RXR treated

lysate
Figure 4 SREBP1 downregulates GH-induced SOCS2 promoter activity and does not compete with STAT5b binding to the promoter. (A, B) BRL-4 cells were co-transfected with the pFAS luciferase reporter plasmid as a control of SREBP1a effect, and with SOCS2 promoter-driven luciferase reporter plasmid (pSOCS2) and SREBP-1a, SREBP-2, or the control vector pCDNA3 at the indicated concentrations. Luciferase activity was measured accordingly. Values are expressed as means \pm SD.

*Indicates significant differences to non-stimulated control transfected cells, \# indicates significant differences to control transfected cells treated with $\mathrm{GH}$.

$* * \mathrm{p}<0.01$. (C, D) BRL-4 cells were treated for $16 \mathrm{~h}$ with $\mathrm{LXR}$ and RXR agonists followed by $10 \mathrm{~min}$ rGH stimulation before lysis. Lysates were used for avidin-Biotin DNA complex assay using DNA fragments of wild type or mutation of response elements for SREBP1 (E-box) or STAT5b as described in the method section. SRE is a control DNA fragment for SREBP1 binding and GHRE is a control for STAT5b binding. A blot for whole cell lysate (WCL) is shown in the bottom panel. (C) GH stimulation was carried out on cells treated with LXR-RXR. (D) GH and LXR-RXR treatments were carried on cells separately and lysates were mixed together accordingly.

treatment was observed in cells overexpressing SREBP1a and SREBP2. We then measured STAT5b mRNA levels after $40 \mathrm{~h}$ of treatment with LXR/RXR combination or after SREBP1a and SREBP2 overexpression. We found a modest decrease in the STAT5b expression in cells treated with LXR/RXR, or cells overexpressing SREBP1a and SREBP2 (Figure 5C). These findings indicate that elevation of SREBP1 and SREBP2 levels may mediate the inhibitory effects of the LXR ligand T0901317 on GH stimulated transcription through inhibition of $S T A T 5 b$ gene expression and by increasing the degradation of STAT5b protein.

\section{Discussion}

In the present study, we demonstrated that LXR activation with the synthetic ligand T0901317 attenuates hepatic GH signaling by reducing STAT5b activity and thereby inhibiting GH induced gene transcription. We also showed that the effects of LXR agonists on GH signaling are mimicked by overexpression of two LXR downstream target genes, SREBP1 and $S R E B P 2$. The inhibitory effects of these lipogenic factors on STAT5b protein levels can partly be explained by their inhibitory effects on $S T A T 5 b$ gene transcription and the enhancement of STAT5b protein degradation.

In this study, we provide several lines of evidence to support the inhibitory activity of the LXR agonist T0901317 on STAT5b activity in hepatocytes. T0901317 treatment significantly reduced GH induction of mRNA levels of STAT5b regulated gene, SOCS2. Likewise, the reporter luciferase activity, driven by the STAT5b response element in the SOCS2 gene promoter, was inhibited by the LXR agonist. Direct measurements of T0901317 effects on STAT5b protein levels, and its DNA binding activity, also show the inhibitory effects. Finally, overexpression of a well-known LXR target gene, SREBP1a, can mimic the effects of LXR ligands in reducing $\mathrm{GH}$ activated transcription and STAT5b levels. The 
A

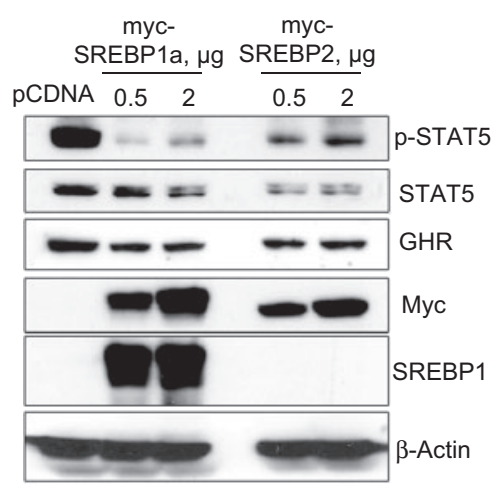

B

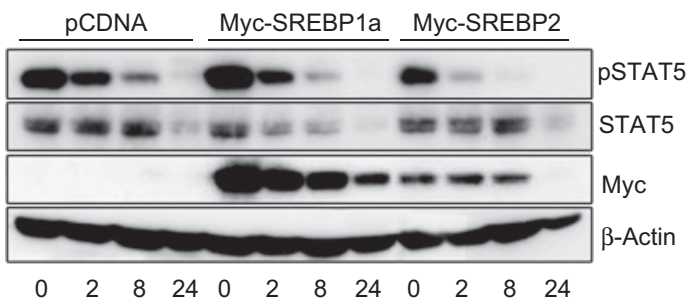

$\mathrm{CHX}$,
$100 \mu \mathrm{g} / \mathrm{m}$

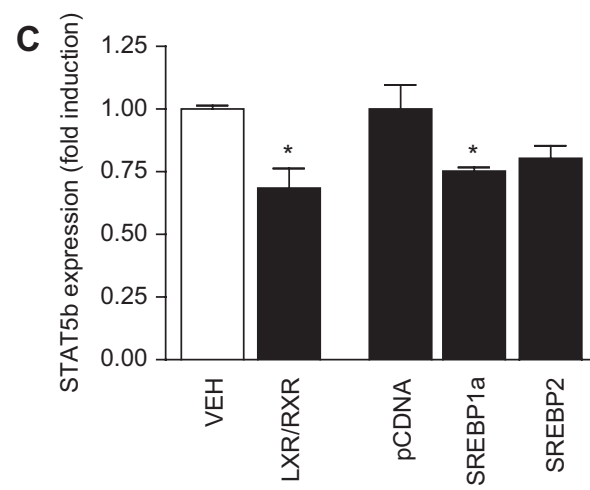

Figure 5 SREBPs downregulate STAT5b activity in liver cells. Myc tagged SREBP1a and SREBP2 proteins were overexpressed in BRL-4 cells followed by measurement of STAT5b protein and mRNA levels. (A) Western blot for phosphorylated and total STAT5b in cells transfected with myc-SRBEP1a, myc-SREBP2 or pCDNA3 control vector. (B) Cycloheximide (CHX) chase experiments were performed after overexpression of SREBP1a and SREBP2 in BRL-4cells. Then stability of STAT5b was measured by following the protein levels by Western blot. (C) Fold change in STAT5b mRNA expression after $48 \mathrm{~h}$ treatment of LXR and LXR agonist and in cells overexpressing SREBP1a and SREBP2. The ribosomal S18 mRNA levels were used as controls and used to normalize the data. Data shown are means \pm SD.

*Indicates a significant difference to control cells. ${ }^{*} \mathrm{p}<0.05$.

CHX chase experiments demonstrated that SREBP1a promotes the degradation of STAT5b. Little is known about the mechanisms that regulate STAT5b turnover. Previous work has implicated proteasomal degradation in the downregulation of activated STAT5a and demonstrated its ubiquitination in the nucleus [19]. Furthermore, a recent in depth proteome screening of ubiquitylation sites, has identified the ubiquitin modification of lysine 567 of the human STAT5b [20]. Proteasomal degradation of STAT5a is modulated by a short motif comprising amino acids $751-762$ of the C-terminal domain [19]. This region is highly conserved in STAT5b, corresponding to amino acids 757-768 [21]. The E3 ubiquitin ligase complex responsible for the ubiquitination of STAT5 in the nucleus remains to be identified. Our data would suggest that the activity of this E3 ligase could be modulated by SREBP1a and SREBP2 activation.

Most of the mechanistic studies presented in this work were performed in rat BRL cells stably transfected with the rat GHR, with the key finding also confirmed in primary human hepatocytes. In primary hepatocytes, we observed that LXR stimulation results in severe downregulation of GH receptor levels parallel to downregulation of STAT5b, suggesting that additional mechanisms exist whereby LXR agonists inhibit GH actions. Liver microarray analysis of human, mice, and rat livers have demonstrated that LXR activation leads to decreased GH receptor mRNA levels, providing the most likely explanation for these effects [2, 22, 23]. We have also made an interesting observation that the GHR protein levels in rat BRL-4 cells, in which its expression is driven by the CMV promoter, were not changed by the LXR agonist treatment, but yet we observed a significant reduction of STAT5b levels. Therefore, the LXR driven mechanisms that target GH signals seem to operate on both the GHR as well as STAT5b. This level of redundancy suggests that GH antagonism may be an important aspect of LXR physiological actions. All data presented in this study concern experiments performed in cultured cells. Therefore, further studies are warranted to evaluate the contribution of $\mathrm{GH}$ on the pharmacological actions of LXR agonist in vivo. Additional studies are also needed to verify whether additional tissue specific actions of LXR involve STAT5b downregulation. Tissue variations in the expression of different LXR isoforms or SREBPs content, as well as differences in the treatment modalities, may influence LXR ligand actions on STAT5b levels. For example, it has been reported that T0901317 treatment in lymphocytes did not affect the induction of STAT5b serine and tyrosine phosphorylation by IL-2 [24].

The demonstration that SREBP1 activation leads to STAT5b downregulation in hepatic cells may have wider implications, as several physiological situations are associated with increased hepatic expression of this factor. Notably, SREBP1 is stimulated by insulin after feeding, which may serve as a mechanism to control GH sensitivity postprandially [25]. Our findings may also have important consequences towards the goal of designing selective LXR agonists with better activity profiles. If indeed, $\mathrm{GH}$ antagonism is a prominent feature of LXR activation, it may be very difficult to separate LXR driven hepatic steatosis from its beneficial actions on insulin sensitivity, as they may be commonly regulated through the inhibition of STAT5b activity. In contrast, LXR agonists with reduced activity towards SREBPs, may still be of use in the treatment of hypercholesterolemia, which may rely on direct regulation of transporters, such as $A B C D A 1$ and $A B C D G 1$, and cholesterol metabolizing genes, such as $7 \alpha$-hydroxylase (CYP7A1) by LXR [1].

In conclusion, the results presented in this paper indicate that LXR agonists downregulate STAT5b protein levels and 
suppresses GHR activity in hepatocytes. Further investigation of the physiological consequences of the LXR/GHR interaction should provide evidence for a better informed use of LXR agonist and may explain some of its effect across a range of metabolic phenotypes.

\section{Acknowledgments}

This work was supported by the Danish Research Council, Vetenskapsrådet (No 2009-3738) and the Novo Nordisk Research Foundation. Fahad Zadjali was supported by a fellowship from Sultan Qaboos University, Oman. Ruyman Santana-Farre was the recipient of pre-doctoral fellowships from the Spanish Ministry of Science and Innovation (MCYT) (SAF2003-02117) and ACIISI-FSE (SE-10/13). Leandro Fernandez-Perez, Gunnar Norstedt and Amilcar Flores-Morales are senior contributors to this work. This work was supported in part by grants to Leandro Fernandez-Perez from MCYT with the funding of the European Regional Development FundEuropean Social Fund (SAF2003-02117 and SAF2006-07824).

\section{References}

1. Wojcicka G, Jamroz-Wisniewska A, Horoszewicz K, Beltowski J. Liver X receptors (LXRs). Part I: structure, function, regulation of activity, and role in lipid metabolism. Postepy Hig Med Dosw (Online) 2007;61:736-59.

2. Loffler M, Bilban M, Reimers M, Waldhausl W, Stulnig TM. Blood glucose-lowering nuclear receptor agonists only partially normalize hepatic gene expression in $\mathrm{db} / \mathrm{db}$ mice. J Pharmacol Exp Ther 2006;316:797-804.

3. Grefhorst A, Elzinga BM, Voshol PJ, Plosch T, Kok T, Bloks VW, van der Sluijs FH, Havekes LM, Romijn JA, Verkade HJ, Kuipers F. Stimulation of lipogenesis by pharmacological activation of the liver $\mathrm{X}$ receptor leads to production of large, triglyceride-rich very low density lipoprotein particles. J Biol Chem 2002;277:34182-90.

4. Rico-Bautista E, Flores-Morales A, Fernandez-Perez L. Suppressor of cytokine signaling (SOCS) 2, a protein with multiple functions. Cytokine Growth Factor Rev 2006;17:431-9.

5. Fan Y, Menon RK, Cohen P, Hwang D, Clemens T, DiGirolamo DJ, Kopchick JJ, Le Roith D, Trucco M, Sperling MA. Liverspecific deletion of the growth hormone receptor reveals essential role of growth hormone signaling in hepatic lipid metabolism. J Biol Chem 2009;284:19937-44.

6. Ichikawa T, Hamasaki K, Ishikawa H, Ejima E, Eguchi K, Nakao K. Non-alcoholic steatohepatitis and hepatic steatosis in patients with adult onset growth hormone deficiency. Gut 2003;52:914.

7. Kalaany NY, Gauthier KC, Zavacki AM, Mammen PP, Kitazume T, Peterson JA, Horton JD, Garry DJ, Bianco AC, Mangelsdorf DJ. LXRs regulate the balance between fat storage and oxidation. Cell Metab 2005;1:231-44.

8. Korach-Andre M, Archer A, Gabbi C, Barros RP, Pedrelli M, Steffensen KR, Pettersson AT, Laurencikiene J, Parini P, Gustafsson JA. Liver X receptors regulate de novo lipogenesis in a tissue-specific manner in C57BL/6 female mice. Am J Physiol Endocrinol Metab 2011;301:E210-22.

9. Kopchick JJ, Andry JM. Growth hormone (GH), GH receptor, and signal transduction. Mol Genet Metab 2000;71:293-314.

10. Vidal OM, Merino R, Rico-Bautista E, Fernandez-Perez L, Chia DJ, Woelfle J, Ono M, Lenhard B, Norstedt G, Rotwein P, Flores-
Morales A. In vivo transcript profiling and phylogenetic analysis identifies suppressor of cytokine signaling 2 as a direct signal transducer and activator of transcription $5 \mathrm{~b}$ target in liver. Mol Endocrinol 2007;21:293-311.

11. Bennett MK, Lopez JM, Sanchez HB, Osborne TF. Sterol regulation of fatty acid synthase promoter. Coordinate feedback regulation of two major lipid pathways. J Biol Chem 1995;270:25578-83.

12. Castrillo A, Joseph SB, Vaidya SA, Haberland M, Fogelman AM, Cheng G, Tontonoz P. Crosstalk between LXR and toll-like receptor signaling mediates bacterial and viral antagonism of cholesterol metabolism. Mol Cell 2003;12:805-16.

13. Sundqvist A, Ericsson J. Transcription-dependent degradation controls the stability of the SREBP family of transcription factors. Proc Natl Acad Sci USA 2003;100:13833-8.

14. Ellis EC, Nilsson LM. The use of human hepatocytes to investigate bile acid synthesis. Methods Mol Biol 2010;640: 417-30.

15. Flores-Morales A, Fernandez L, Rico-Bautista E, Umana A, Negrin C, Zhang JG, Norstedt G. Endoplasmic reticulum stress prolongs GH-induced Janus kinase (JAK2)/signal transducer and activator of transcription (STAT5) signaling pathway. Mol Endocrinol 2001;159:1471-83.

16. Albers M, Blume B, Schlueter T, Wright MB, Kober I, Kremoser C, Deuschle U, Koegl M. A novel principle for partial agonism of liver $\mathrm{X}$ receptor ligands. Competitive recruitment of activators and repressors. J Biol Chem 2006;281:4920-30.

17. Han C, Wang J, Li L, Zhang Z, Wang L, Pan Z. The role of insulin and glucose in goose primary hepatocyte triglyceride accumulation. J Exp Biol 2009;212:1553-8.

18. Wood TJ, Sliva D, Lobie PE, Goullieux F, Mui AL, Groner B, Norstedt G, Haldosen LA. Specificity of transcription enhancement via the STAT responsive element in the serine protease inhibitor 2.1 promoter. Mol Cell Endocrinol 1997;130: 69-81.

19. Chen Y, Dai X, Haas AL, Wen R, Wang D. Proteasomedependent downregulation of activated Stat5A in the nucleus. Blood 2006;108:566-74.

20. Wagner SA, Beli P, Weinert BT, Nielsen ML, Cox J, Mann M, Choudhary C. A proteome-wide, quantitative survey of in vivo ubiquitylation sites reveals widespread regulatory roles. Mol Cell Proteomics 2011 Oct;10(10): M111.013284. Epub 2011 Sep 1.

21. Lin JX, Mietz J, Modi WS, John S, Leonard WJ. Cloning of human Stat5B. Reconstitution of interleukin-2-induced Stat5A and Stat5B DNA binding activity in COS-7 cells. J Biol Chem 1996;271:10738-44.

22. Stulnig TM, Steffensen KR, Gao H, Reimers M, Dahlman-Wright K, Schuster GU, Gustafsson JA. Novel roles of liver X receptors exposed by gene expression profiling in liver and adipose tissue. Mol Pharmacol 2002;62:1299-305.

23. Kotokorpi P, Gardmo C, Nystrom CS, Mode A. Activation of the glucocorticoid receptor or liver $\mathrm{X}$ receptors interferes with growth hormone-induced akr1b7 gene expression in rat hepatocytes. Endocrinology 2004;145:5704-13.

24. Geyeregger R, Shehata M, Zeyda M, Kiefer FW, Stuhlmeier KM, Porpaczy E, Zlabinger GJ, Jager U, Stulnig TM. Liver X receptors interfere with cytokine-induced proliferation and cell survival in normal and leukemic lymphocytes. J Leukoc Biol 2009;86:1039-48.

25. Bennett WL, Ji S, Messina JL. Insulin regulation of growth hormone receptor gene expression. Evidence for a transcriptional mechanism of downregulation in rat hepatoma cells. Mol Cell Endocrinol 2007;274:53-9. 